\title{
Otium, re-création, conversion : les vacances peu orthodoxes de Henry Edward Manning, archidiacre de Chichester (1838-1848)
}

Otium, Re-creation, Conversion: the Unorthodox Vacations of Edward Henry

Manning, Archdeacon of Chichester (1838-1848)

Jacqueline Clais-Girard

\section{OpenEdition \\ Journals}

Édition électronique

URL : http://journals.openedition.org/rfcb/3629

DOI : $10.4000 /$ rfcb.3629

ISSN : 2429-4373

Éditeur

CRECIB - Centre de recherche et d'études en civilisation britannique

Édition imprimée

Date de publication : 1 mars 2013

ISBN : 2-911580-37-0

ISSN : 0248-9015

Référence électronique

Jacqueline Clais-Girard, "Otium, re-création, conversion : les vacances peu orthodoxes de Henry Edward Manning, archidiacre de Chichester (1838-1848)», Revue Française de Civilisation Britannique [En ligne], XVIII-1 | 2013, mis en ligne le 01 mars 2013, consulté le 24 mars 2020. URL : http:// journals.openedition.org/rfcb/3629; DOI : https://doi.org/10.4000/rfcb.3629

Ce document a été généré automatiquement le 24 mars 2020

Revue française de civilisation britannique est mis à disposition selon les termes de la licence Creative Commons Attribution - Pas d'Utilisation Commerciale - Pas de Modification 4.0 International. 


\title{
Otium, re-création, conversion : les vacances peu orthodoxes de Henry Edward Manning, archidiacre de Chichester (1838-1848)
}

\author{
Otium, Re-creation, Conversion: the Unorthodox Vacations of Edward Henry \\ Manning, Archdeacon of Chichester (1838-1848)
}

Jacqueline Clais-Girard

1 Fils d'un riche marchand, député conservateur et un temps gouverneur de la Banque d'Angleterre, Henry Edward Manning, le benjamin de huit enfants, est le seul à recevoir une éducation universitaire, car on le destine à une carrière ecclésiastique. Une réussite brillante aux examens en novembre 1830 lui permet d'espérer une carrière politique plus conforme à ses goûts. Lorsque la ruine de son père vient mettre un terme à ce projet, il présente sa candidature à un poste de fellow à Merton, tout en avouant au warden que, conscient de ne pas avoir la vocation, il a décidé de ne pas être prêtre ${ }^{1}$. Il passe l'été 1831 seul dans la propriété familiale de Coombe Bank, avant qu'elle ne soit vendue. Frustré, désœuvré, déprimé, il trouve refuge à Trent Park, la propriété des Bevan, une famille amie dont le fils est un ancien condisciple de Harrow. Lors d'un précédent séjour, il était tombé sous l'influence de la très pieuse sœur ainée de Robert, celle, sans doute, qui a le mieux cerné sa personnalité complexe ${ }^{2}$. Miss Bevan va lui démontrer que les ambitions célestes ne lui sont pas fermées ${ }^{3}$, et qu'il est possible de concilier ambition et conscience; s'opère alors, sous son influence, une sorte de première conversion. Le 24 novembre, elle écrit dans son journal: 'H.M. is in the Hands of One Who can guide by His counsel[...] Who knows but that after being tempest-tossed for a season he may seek the service of his Master?'4

2 Cet été-là, en tout cas, la religion prend sur lui un ascendant qu'elle n'avait encore jamais eu; alors que, le 13 juin 1831, il écrivait à son beau-frère : 'I am splenetic, sick, savage, sour, rabid, indolent, useless, and ill at ease ${ }^{15}$, six mois plus tard, il a fait son choix, il sera prêtre. Dès lors, les choses vont très vite : fellow de Merton en avril 1832, diacre en 
décembre, le jeune Henry accepte en janvier 1833 de remplacer Henry Wilberforce comme vicaire auprès du révérend John Sargent à Lavington, dans le Sussex, avec l'intention bien arrêtée de revenir à Oxford avant l'été. D'abord uniquement chargé de la petite église d'Upwaltham, Henry Manning se voit offrir à la mort du révérend Sargent son bénéfice, qui regroupe les trois paroisses de Lavington, de Graffham et d'Upwaltham. Ordonné le 9 juin, il épouse le 7 novembre Caroline, la troisième fille de la maison, et devient le recteur d'une paroisse modèle qui ne compte guère plus de 800 âmes : " À 25 ans marié, renté, haut placé, Manning était dans la plus enviable des positions " ${ }^{6}$. Même pour un homme de petite santé, la tâche n'était pas écrasante et lui laissait le temps de lire, de se cultiver ${ }^{7}$, et de réfléchir au sacerdoce à partir de la pratique qu'il découvrait et de l'atmosphère théologique du moment - c'est en juillet 1833 que commence le Mouvement d'Oxford.

3 S'il a embrassé de tout cœur la piété et les pratiques de dévotion évangéliques en vigueur chez les Bevan et dans la famille Sargent, il n'a pas adopté leurs opinions doctrinales. Son bagage théologique tient en quelques phrases: 'When I came to Lavington in 1833 I believed, as I always did, in baptismal regeneration: I had no view of the Sacrament of the Body and Blood of Christ and no idea of the Church. You sent me in the year 1834 to Hooker to learn the doctrine of the Real Presence's, écrira-t-il à Samuel Wilberforce en 1850. Comme son beau-père, il visite régulièrement ses paroissiens, mais il a besoin, pour ne pas se sentir importun, de se croire investi d'une mission divine et porteur d'un message divin'. C'est ainsi qu'il adopte très vite le principe de la succession apostolique, dans lequel cette mission divine trouve son origine et sa justification. Son sermon de 1835, The English Church, Its Succession and Witness for Christ, qui magnifie le sacerdoce, fait écho aux premiers tracts de John Henry Newman, qu'il a d'ailleurs proposé de distribuer dans le Sussex ${ }^{10}$. De même, c'est la question du message divin qui l'amène à la tradition apostolique. Son ministère pastoral le rend désireux de trouver une règle qui permette de certifier les doctrines chrétiennes ${ }^{11}$. Les évangéliques mettent l'accent sur le jugement privé, les catholiques, sur l'interprétation de l'Église, et les anglocatholiques, sur l'Écriture et la Tradition - deux témoins d'une seule vérité -, une position que les évangéliques vont attaquer comme trop romaine, et les catholiques, comme trop proche du jugement privé. C'est en tout cas celle que-défend Manning dès 1836 dans une lettre au British Magazine signée 'a Catholic Priest', et qu'il précisera dans The Rule of Faith, son sermon de 1838, qui forme, avec l'appendice publié suite aux attaques des évangéliques, un traité assez complet de la règle de la foi.

Certains de ses amis s'étaient inquiétés de le voir «s'enterrer" dans une cure de province. Peu avant qu'il ne reçoive les ordres majeurs, Edward Twistleton lui écrivait: ‘ I prophesy that you will find the retirement of a village curacy highly charming at first - for you know you have naturally a mixture of the recluse in your disposition - but I hope you will quietly look forward to a more active sphere of exertion, and will not suffer your energies to lie dormant $^{12}$. Plus que l'ambition, pourtant, ce sont les circonstances qui vont lui faire choisir une sphère plus active. En effet, cette vie de "learned leisure and earthly happiness 'que décrit son biographe E. S. Purcell ${ }^{13}$ prend brutalement fin à la mort de Caroline, le 24 juillet 1837; Manning découvre alors que le but de la vie n'est pas d'être heureux, mais d'être utile, et se jette à " cœur» perdu dans le travail ${ }^{14}$. Avec son évêque, il défend l'indépendance de l'Église par rapport au pouvoir civil ; secrétaire du comité diocésain pour l'éducation, il lance avec quelques amis un plan pour l'enseignement primaire confessionnel; doyen rural de Midhurst, puis archidiacre de Chichester, il s'emploie à remettre de l'unité dans les paroisses du diocèse. Sa réputation grandit si 
rapidement que la défection de Newman va le placer sur le devant de la scène anglicane : en toute première ligne, et en plus grand danger, en raison de son évolution personnelle. C'est justement cette évolution qu'il peut être intéressant de mettre en parallèle avec les voyages qu'il effectue sur le continent de 1838 à 1848 en France, en Belgique, en Allemagne, et en Italie. Souvent très brefs, ces voyages sont, la plupart du temps, destinés à récupérer de sa fatigue physique et nerveuse, voire de la maladie consacrés, donc, à la re-création (re-creatio) plus qu'à la récréation (otium) -, car une aussi spectaculaire ascension se fait forcément au détriment d'une santé qu'il ne ménage guère ; mais ne répondent-ils pas aussi à une aspiration qu'il ne s'avoue pas ?

\section{Premier séjour en terre hérétique}

Dès l'été 1834, Henry Wilberforce avait rapporté à Newman les progrès qu'il constatait chez celui qui était maintenant son beau-frère ${ }^{15}$; ceux-ci sont confirmés par William Gladstone, qui note dans son journal qu'en l'espace de deux ou trois ans, Manning est devenu ardemment anglican. Mais l'évolution de sa foi ne s'arrête pas là. Apprenant que Manning est parti passer l'hiver 1838-1839 en Italie, l'évêque de Londres aurait dit qu'il le croyait à Rome depuis son dernier volume de sermons ! ${ }^{16}$ Pourtant, ce premier voyage à Rome n'est pas de son fait ; ce sont ses amis, inquiets de son état de santé, qui le lui ont imposé. Souffrant de violentes crises d'asthme, Manning n'a guère envie de voyager, et refuse d'accompagner Gladstone lorsque son ami part en août « se reposer les yeux » à Rome et en Sicile. En octobre, pourtant, il doit céder aux instances de son entourage, et part le retrouver en compagnie du jeune Arthur Harrison, qui va lui aussi à Rome pour raisons de santé. Arrivés le lundi 10 décembre, ils consacrent, comme Newman et Hurrell Froude cinq ans plus tôt, leur toute première visite à Nicolas Wiseman, recteur de l'English College; sans doute Manning ne se vante-t-il pas de l'avoir attaqué deux ans plus tôt dans le British Magazine. Ils se rendent ensuite à Saint-Pierre, où, après avoir admiré l'édifice, ils entendent les vêpres. Le jeudi 13 décembre, ils passent l'après-midi au Vatican, où le peintre Joseph Severn, qui réside alors à Rome il y fait aussi fonction de consul - leur fait admirer les statues. Le dimanche 23, Manning et Gladstone assistent ensemble à la messe. La semaine précédente, trouvant les sermons anglicans décidément trop ennuyeux, Manning est allé presque tous les jours écouter le nouvel évêque d'Alger, Alphonse Dupuch, prêcher à Saint-Louis-desFrançais. Le 29 décembre, Gladstone, Manning et Harrison se rendent de nouveau à l' English College pour y entendre la messe ${ }^{17}$. Aucune autre rencontre n'est mentionnée en janvier, sans doute en raison du retour de Gladstone en Angleterre, mais le 21, Wiseman emmène Manning à Sainte-Agnès-hors-les-Murs assister à la bénédiction des agneaux dont la laine sert à fabriquer les palliums. Désormais seul à Rome, Manning est studieux : il prend des cours de français et d'allemand - 'Study hard at your Deutsch', lui a conseillé son ami S. F. Wood - et lit attentivement le livre de Gladstone, The State in its Relations with the Church (1839), sur lequel il doit donner son avis. Par ailleurs, il se lie d'amitié avec John Sterling, un jeune pasteur ami de Thomas Carlyle (qui passe lui aussi l'hiver à Rome pour raisons de santé), et devient membre du Sterling Club, un cercle très huppé qui doit son nom autant à la qualité de ses membres qu'au patronyme de son fondateur. John Sterling fait à Richard Trench un portrait flatteur - et prophétique - de son nouvel ami, qui confirme, s'il en était besoin, qu'il est bel et bien perdu pour les évangéliques, et conclut: 'I conceive him to be, in his own place and generation, one of the 
most practically efficient and energetic men I have ever known, and in a state of freer and more fluent life in the ecclesiastical polity he would rise high and do considerable things ${ }^{18}$.

\section{Le temps du doute}

6 Gladstone, qui est arrivé à Londres fin janvier, mettra deux mois pour lire les sermons de son ami, qui ont pourtant fait grand bruit l'année précédente - retard dont la politique n'est pas seule responsable, puisque le 14 juin, il lui annonce ses fiançailles ${ }^{19}$. Manning est déjà rentré à Lavington. Pourtant, il n'assistera pas au mariage de son ami célébré le 25 juillet 1839, deux ans presque jour pour jour après la mort de Caroline. De retour de Rome, il ne semble pas particulièrement attiré par le catholicisme, même s'il reconnaît volontiers la qualité des prédicateurs catholiques qu'il est allé, sur les instances de Samuel Wood, écouter avec beaucoup d'application et, sans doute, de plaisir. Il apprécie les éléments catholiques présents dans l'Église romaine, qui lui manquent dans l'Église d'Angleterre, mais le système romain lui paraît corrompu. À propos des conversions au catholicisme dont il a connaissance, il écrit le 23 mars : 'The occasional conversion of a serious person hides the taint of the system '20; et, à l'automne 1839 , on le voit consulter Newman ${ }^{21}$ pour savoir comment traiter le cas des personnes tentées par "l'hétérodoxie " catholique. En décembre 1840, quelques semaines avant que Gladstone n'entre au Conseil Privé, Manning a été nommé archidiacre de Chichester, à la grande satisfaction de Julius Hare, son homologue de Lewes. Sa réputation est telle que Phillpotts, le perspicace évêque d'Exeter, va déclarer cette année-là: 'There are three men to whom the country has mainly to look in the coming years Manning in the Church, Gladstone in the State, and Hope [afterwards Mr. Hope-Scott, Q. C.] in the Law ${ }^{22}$. Manning travaille avec acharnement au service de son Église - sermons et mandements, mise en place d'un enseignement primaire confessionnel, création d'évêchés coloniaux, lettres ouvertes contre la Commission ecclésiastique et la suppression des chanoines; collaboration politique avec Gladstone, nommé secrétaire d'État, puis ministre... Les succès s'enchaînent, mais les épreuves ne lui sont pas épargnées : Emily Wilberforce, la sœur aînée de Caroline, meurt le 10 mars 1841, et Mrs Sargent doit le quitter pour aller tenir le ménage de Samuel Wilberforce, alors archidiacre dans le Surrey. C'est dire que Manning suit d'assez loin le Mouvement d'Oxford, avec lequel on l'identifie pourtant, puisque le Record le décrit comme l'un des plus connus et des plus déterminés parmi les tractariens. Manning fera d'ailleurs état de certaines divergences avec Newman: 'In some things I thouroughly agree with Newman, in some things partially, in some not at all'23.

7 Or, après sept années de progrès triomphants, le Mouvement commence à connaître déboire sur déboire. Peu après la condamnation du Tract XC (1841), Newman s'est retiré à Littlemore ; deux ans plus tard, il résigne son bénéfice de St Mary's; enfin, le journal des tractariens, le British Critic, devenu très impopulaire, est suspendu en octobre 1843. Ce même mois, Newman écrit à Manning, qui lui a demandé de s'expliquer: 'I have resigned St Mary's because I think the Church of Rome the Catholic Church and ours not a part of the Catholic Church, because not in communion with Rome'24. Cette lettre a pour premier effet de réveiller les préjugés et la défiance de Manning à l'égard de l'Église romaine ; elle lui révèle brutalement dans quelle direction il s'est inconsciemment aventuré. C'est ainsi qu'il prêche à St Mary's, dans la chaire même de Newman, un sermon résolument anti-romain pour l'anniversaire du Guy Fawkes' Day. Même s'il est resté un « témoin du 
dehors ", Manning se dit blessé par cette défection: 'He seems to tell me that my only stay through six years of sorrow, weariness and solitude is a shadow [...] God be thanked, it does not shake me but it is like a chill on a wound under which one suffers to the very quick'25; et le 21 décembre, il lui écrit:"My dear Newman, do not suspect me as an empty pretender if I say that the only thing that has kept me up in this last six years and more of trial, and the only thing I look for until death is to save the Church in which I was born again. Doubtful thoughts about it are dreadful - and seem to take all things from $m e^{226}$. Il ne peut pas douter, il ne veut pas douter.... Comment douter de l'Église qui l'a soutenu au cours de toutes ces années d'épreuves?

8 Juin 1844 voit la publication du livre de William G. Ward, The Ideal of a_Christian Church. Manning n'apprécie guère qu'on lui propose l'Église romaine pour modèle, mais il doit reconnaître la justesse du diagnostic que Ward pose sur les maux dont souffre l'Église d'Angleterre ${ }^{27}$. Sa lettre à Gladstone, le 13 juillet, se termine sur ces mots: 'I have heavier thoughts for the Church of England than I had and I fear truer'28. Inquiet à l'idée de se trouver en désaccord avec son évêque - le nouvel évêque de Chichester est très hostile aux tractariens -, il demande à son ami quelle attitude adopter si son mandement contient des jugements auxquels il ne saurait souscrire: 'This seems to me a question of practice such as you are well versed in!' écrit-il, mais elle est symptomatique des doutes qu'il nourrit à l'égard de la hiérarchie ${ }^{29}$. Alors qu'un mois plus tôt, il écrivait à son ami William Dodsworth : 'If I doubted, I would cease to speak and act till I was decided either for England or Rome ${ }^{\prime 30}$, il est évident que, s'il n'a pas encore la certitude que l'Église de Rome est la véritable Église, il doute désormais que l'Église anglicane soit une branche de l'Église catholique. S'éloigner, prendre du recul, lui permettrait peut-être de voir où il en est. Manning choisit d'aller en Normandie, région proche que les Anglais apprécient particulièrement, région qui possède aussi un important patrimoine architectural religieux. Parti le 25 septembre, sitôt après la visite du Dr Gilbert, il est le lendemain soir à Dieppe, où il retrouve Henry Wilberforce. Le 27, il est à Rouen ; le 30, à Bayeux, à Saint-Lô, et à Coutances ; le mardi 1er octobre, à Granville, à Avranches et à Pontorson ; le mercredi 2, au Mont-Saint-Michel et à Saint-Malo. Le vendredi 4, il se rend à Jersey et à Guernesey; il arrive le soir à Plymouth, d'où il gagne Exeter par le coche, puis Reading par le train. On reste perplexe devant cette espèce de marathon qu'il décrit à Gladstone : 'Since I wrote to you I have been ten days in Normandy. I went from Rouen by Caen, St Lo, Coutances, Avranches to Mont S. Michel \& St Malo, and made a very pleasant sally' ${ }^{31}$.

9 A-t-il au moins réussi à se changer les idées? Son mandement de 1845 pourrait le laisser supposer: 'Fifteen years, certainly adverse to the external clothing of the Church, have passed over us [...] And yet, despite of all this, there has arisen within the Church [...] an energy and power of expansion never seen before ${ }^{\prime 32}$. Au moment où ce mandement est prononcé, Manning éprouve de nouveau un immense besoin de secouer un joug qu'il trouve de plus en plus pesant. Pressent-il que ce renouveau qu'il met en avant n'est qu'un trompe-l'œil, et que l'avenir est sombre? Richard Church dira plus tard que les conversions individuelles se firent plus nombreuses à partir de l'été 1845 : 'It was not till the summer that the first drops began to fall [...] Then through the autumn and the next year friends whose names and forms were familiar to Oxford one by one disappeared and were lost to $i t$; fellowships, curacies and intended careers were given up ${ }^{\prime 33}$. Le 14 août, à peine rentré d'Easton, où son beau-frère, George D. Ryder, est recteur, Manning écrit à Gladstone: ' These things make my heart ache, for I have enough on me in other ways. I do not shrink or repine: but I sigh for rest both of body \& of spirit. Sometimes I feel deeply sorrowful ${ }^{34}$. Il projette de faire le voyage à Paris qu'il n'a pu faire l'année précédente. Le prétexte est 
tout trouvé : les Ryder vont quitter l'Angleterre et se rendre sur le continent pour la santé de Sophia, la sœur de Caroline, emmenant avec eux trois de leurs enfants et Sophy, la sœur de George. Ce dernier a entrepris un livre qui doit prouver que Rome a failli et que l'Église d'Angleterre représente l'Église primitive, et il compte se mettre sérieusement au travail pendant son séjour.

Le 11 septembre, Manning fait route avec l'archidiacre Charles Marriott jusqu'au Havre, où William Dodsworth le rejoint ainsi que George et Sophia Ryder. Le samedi 13, ils remontent la Seine jusqu'à Paris et, le dimanche 14 septembre, il dîne avec l'évêque Luscombe $^{35}$ et l'archidiacre Keating. S'ensuivent trois jours de visites de chapelles et d'églises dans un Paris qu'il n'a pas revu depuis dix-sept ans: le lundi, la SainteChapelle, Notre-Dame et Saint-Denis ; le mardi, les vieux couvents du faubourg SaintGermain; le mercredi, la Chapelle expiatoire - construite sur l'emplacement du cimetière de la Madeleine où furent inhumés Louis XVI et Marie-Antoinette et les guillotinés jusqu'en 1794 -, le Louvre, enfin Saint-Germain-des-Prés, Saint-Sulpice et son séminaire ${ }^{36}$. À en croire Sophy, les Ryder auraient été surpris de le voir Manning assister à tant d'offices catholiques, comme elle fut elle-même surprise - et néanmoins ravie - de se voir discrètement offrir un traité de dévotion à Marie ${ }^{37}$. Le 18 septembre, leurs chemins se séparent : les Ryder continuent en direction de Fontainebleau, tandis que Dodsworth et Manning repartent pour Rouen. Le 21, ils sont à Southampton, et le lendemain, dimanche 22 septembre - après onze jours de vacances -, Manning est à Lavington pour l'office du matin.

11 Quelques jours avant son départ, William G. Ward et sa jeune femme s'étaient convertis au catholicisme ; quinze jours après son retour, il apprend - par une lettre personnelle - la conversion de Newman. D'Allemagne, Gladstone écrit à sa femme: 'Of all the men we have in the Church of England Manning is the one to take the lead - he has not exactly Newman's peculiar gifts, but he has one which for a long time [...] I have not been able to find in Newman or his immediate friends - namely wisdom ${ }^{\prime 38}$. Manning, qui va peu à Oxford et ne prend pas part aux conflits locaux, ne saurait vraiment succéder à Newman; il n'en a d'ailleurs nulle envie. Malgré tout, on va le considérer désormais comme le chef de file de la "haute Église» (high Church). La formule magique 'credo in Newmanum' est remplacée par 'safe as Manning'; on ne sait pas encore qu'il est ébranlé. Gladstone insiste pour qu'il réfute le dernier livre de Newman, An Essay on the Development of Christian Doctrine (1845), mais il ne pourra pas le faire. Il s'en expliquera des années plus tard: 'I had found the Rule, but not the Judge'39. Il se voit forcé de réexaminer ses notions d'unité et d'infaillibilité. S'ensuit un temps de silence, où Rome lui apparaît plus près de la vérité que l'Église d'Angleterre. Fait-il preuve de sagesse quand il refuse le poste de second aumônier de la reine, laissé libre par la nomination de Samuel Wilberforce comme évêque, ou son refus a-t-il des raisons moins avouables? Celui qui déclarait à Robert Wilberforce à la fin de l'année 1845: 'Nothing can shake my belief in the presence of Christ in our Church and Sacraments. I feel incapable of doubting it ${ }^{40}$, écrit à ce même correspondant six mois plus tard, sous le sceau du secret: 'Tho' not therefore Roman, I cease to be Anglican' ${ }^{31}$. Coïncidence troublante, cette lettre suit de quelques jours la conversion à Rome de Sophy, George et Sophia Ryder, dont il n'a évidemment pas connaissance. 


\section{« Retraite spirituelle en contrées catholiques »}

Le dernier voyage de Manning à Rome en tant qu'anglican est le seul qui soit présenté par Purcell comme 'a spiritual retreat in Catholic countries' ${ }^{42}$. Manning est tombé gravement malade au début de 1847 : sa santé n'a pas résisté au surmenage, ni surtout à la crise intellectuelle et spirituelle qu'il traverse maintenant depuis des années : 'For the last eight years I have been labouring to keep people from the Roman Church [...] During the last four years the effort and anxiety to retain friends in the English Church has perceptibly affected my health; and I can trace I think the beginning of illness last year to that cause', écritil à C. J. Laprimaudaye - son vicaire depuis le 15 janvier $1846^{43}$. Confronté pendant près de trois mois à la maladie qui a emporté sa jeune femme dix ans plus tôt, il a profité de ce repos forcé pour lire les Pères de l'Église et, surtout, le traité de Melchior Cano, De Locis Theologicis (1562) : s'il doit y avoir révélation, il faut qu'il y ait un enseignant et un juge, et ce juge doit être une personne divine, le Saint-Esprit, habitant le corps de l'Église et ses différents membres. Craignant pour son salut, Manning a fait le 18 mars une confession générale à son vicaire. Il doit maintenant cesser tout travail pendant quelque temps et s'exiler, comme le lui a recommandé son médecin ${ }^{44}$. Sa mère meurt au moment où commence sa convalescence, mais il n'est pas question qu'il aille à son enterrement, ni qu'il se rende en Écosse bénir la chapelle que Gladstone vient de faire restaurer ${ }^{45}$; il doit aller vers le sud : 'Dr. Ferguson [...] wishes me to go slowly southward and reach Italy about October', écrit-il à Robert Wilberforce le 9 juin ${ }^{46}$. Malgré les craintes de Laprimaudaye, il a choisi d'aller en Italie, déclinant la proposition moins « dangereuse » de Lord Campden, qui l'invitait à Madère. A-t-il trouvé les réponses aux questions qu'il se posait, ou veut-il tout simplement se laisser guider ? 'Tomorrow by the will of God I go forth, it may be for a year, it may be for ever. I feel to be in His hands. I know not what is good for myself ${ }^{47}$. Au soir de sa vie, il écrira simplement: 'In this state of self-mistrust and fear of going wrong, I went abroad'48.

13 Manning va donc se diriger lentement vers l'Italie: il compte prendre son temps, puisqu'il prévoit cinq mois de voyage, avec des étapes dans une ville d'eaux et dans de hauts lieux du catholicisme. Le 6 juillet, il part en compagnie d'Henry Wilberforce ; ils passent par Bruges, Gand, Louvain, Anvers, Liège et Malines, où Manning doit rencontrer Ignaz von Döllinger. Le 19, il est en Allemagne : Aix-la-Chapelle, Cologne, Bonn, Coblence, Francfort, puis Hombourg - ville protestante 'stripped of outward christianity', où il prend les eaux - et enfin Mayence, où il doit retrouver le 28 août son ami Dodsworth. De Hombourg, Manning écrit le 18 août à Gladstone:

My plans are to go by Basle, Lucerne and the St. Gothard to the lakes and Milan, I hope to see Ravenna. My directions will be until the $1^{\text {st }}$ September, Basle, until the $15^{\text {th }}$ Como, until October $1^{\text {st }}$, Milan then Florence and about the middle of November, Rome, Please God. I have been at this place nearly four weeks, drinking the water, and I trust and believe with real benefit. I feel both stronger and better. ${ }^{49}$

Ces beaux projets ne se réaliseront pas: le 9 septembre, il tombe malade à Lucerne, et doit changer ses plans, gâchant du même coup les vacances italiennes de Dodsworth. $\mathrm{Au}$ lieu de Côme, ce sera l'Angleterre : il repart pour Londres. Après trois semaines de repos complet, il reprend la route du sud, cette fois en compagnie de sa sœur Caroline et de son beau-frère, le colonel Austen. Le 3 novembre, il est à Nice, qu'il quitte le 22 pour l'Italie : l'air y était trop vif. Pendant ces trois semaines, il s'est fait une idée assez précise de la dévotion populaire: 'Here at last was really effective successful religion [...] a sad contrast to our evensong where everyone so far as I saw sat through their prayers ${ }^{50}$. Le 27 
novembre 1847, il est à Rome, où il parvient par bateau de Livourne. Malgré ses problèmes de santé, il n'arrive guère que deux semaines après la date initialement prévue. À Gladstone, il écrit que, si tout lui rappelle leur séjour effectué dix ans plus tôt, l'ambiance est très différente: 'Outwardly Rome is unchanged - the streets, shops, pavements, heaps of dirt not yet taken away. But morally and inwardly there is a vast change: $a$ very visible increase of intelligence and energy, with free public expression in word and writing' ${ }^{51}$. Responsable de ce changement: Pie IX, le nouveau pape, qui a amnistié, réformé et libéralisé, sans se douter qu'il mettait en péril sa propre position; un risque dont Manning se montre très conscient: 'I [...] am afraid that anything which upsets an existing order, instead of recalling it to its first idea, only clears a field for confusion'52.

À défaut d'être tout à fait la même, la bonne société londonienne est toujours là. Chez les Herbert, Manning rencontre Mr et Mrs Bracebridge accompagnés de Florence Nightingale - une Florence âgée tout juste de 27 ans qui, fort perspicace, écrit à son père: 'He [Manning] will make an easy transit from the Via della Croce to the Convento della Santissima Croce in Gerusalemme and occupy the wide and sunny cell just left by Newman' ${ }^{53}$. Manning n'a fait que croiser Newman, qui rentre en Angleterre après avoir achevé son noviciat d'Oratorien, mais il fréquente d'autres convertis récents comme Ambrose St John et Frederick Bowles, et donne libre cours à son besoin d'en savoir plus sur le culte catholique et ses dévotions : il prend des notes pendant les sermons, dresse le plan des églises, et discute avec des prêtres, des moines et des religieux, montrant un intérêt tout particulier pour les jésuites et les passionistes ${ }^{54}$. Il fait ce que Newman se défend, dans une lettre à un ami, d'avoir jamais fait ${ }^{55}$.

Du 27 novembre au 11 mai 1848, date à laquelle il repart pour l'Angleterre via Assise et Milan, Manning aura de nouveau séjourné près de six mois à Rome; il a été reçu en audience privée par le pape le 9 avril - lui remettant à cette occasion le pamphlet de sir Charles Trevelyan sur la famine en Irlande - et une deuxième fois le jour de son départ. L'absence de commentaires immédiats sur ces deux rencontres laisse à penser qu'il en garde un souvenir mitigé - peut-être en raison de l'ignorance du Saint-Père concernant l'Église d'Angleterre. L'aspect religieux de son voyage se double d'un intérêt politique. Les dernières semaines ont été riches en événements : en Italie, comme partout en Europe, c'est le «Printemps des peuples». On crie "Vive Pie IX!» à Rome dans l'euphorie du printemps - euphorie qui ne va pas durer. Manning a suivi ces événements avec espoir: 'It is wonderful to see the Catholic Church in America, France \& Italy distinctly of the progress and popular party: indeed in many ways at the head of $i t^{556}$; et il se souviendra toute sa vie des paroles du Père Gioacchino Ventura à Pie IX: 'Let not Your Holiness look to the sovereigns of Europe who are shadows who may vanish within the year, but to the peoples who are realities and last for ever ${ }^{157}$. La confusion qui règne en Europe - et le laisse incertain quant à son itinéraire de retour - ne l'empêche pas de désirer voir la Lombardie 'in its first days of freedom'. Il repart de Naples pour Livourne en bateau ; de là, il se rend à Florence, puis à Ravenne, puis revient à Florence pour gagner Pavie et Milan. Cette dernière, qu'il n'a pu visiter à l'aller, est le véritable but de ce périple : c'est la ville d'Ambroise, d'Augustin et de Charles Borromée. Auprès du tombeau de ce dernier, Manning croit entendre un appel, car les ultimes paroles de l'Évangile 'et erit unum ovile et unus pastor' ont retenti à son oreille d'une manière toute nouvelle. "Un seul troupeau, un seul pasteur » : serait-ce là le signe qu'il attendait ?58 


\section{La conversion}

Le 18 juin 1848, après presque un an d'absence, Manning est de retour à Londres chez sa sœur, Mrs Carey, 44 Cadogan Square. Il semble que les effluves de Rome se soient dissipés : serait-il dégrisé ? Gladstone, qui le rencontre longuement le 9 juillet, le trouve confirmé dans son allégeance à l'Église d'Angleterre et enclin à insister sur sa catholicité, mais n'est-il pas influencé par la décision de Manning de défendre de son mieux la position de l'Église anglicane dans l'affaire Hampden ${ }^{59}$ Très inquiet des conséquences que cette affaire pourrait avoir, Manning ne peut se permettre de la traiter avec légèreté dans son mandement. Il explique à George Moberly que ses opinions n'ont pas changé : il est persuadé de l'hétérodoxie de Hampden, mais il lui faut plaider la cause de l'Église d'Angleterre, sinon comment servir une Église qu'il ne pourrait défendre $?^{60}$ Jusqu'au bout, il fera preuve de loyauté. Lorsque l'enseignement confessionnel, pour lequel il s'est beaucoup impliqué, est menacé d'ingérence par le gouvernement en 1849, il travaille avec Gladstone pour obtenir un compromis acceptable par le clergé, et fait de son mieux pour qu'il soit accepté. À cette occasion, F. D. Maurice remarque: 'There was one man in that room who can save the Church from its confusion if he has it in his mind to do so. This was Manning. Mr. Anderson agreed with me, but had some doubts about his will [...] His power with the clergy is very great, greater certainly than that of any man living'61. C'est précisément cette influence qui rend la décision de Manning si difficile à prendre. Craignant d'être le jouet d'une illusion, il veut attendre d'avoir la conviction qu'il fait la volonté de Dieu :

I have felt and do feel an overwhelming fear lest I should be under an illusion. I know of nothing else which weighs with me but this, lest I contradict the will of my Lord. On St. Andrew's Day I offered myself as I have again and again, and never so often as in Rome, to follow on the spot if only I can have, not sign or token, but the conviction of a moral agent that it is the will of my Lord. ${ }^{62}$

L'affaire Gorham va lever ses derniers doutes. Qu'un tribunal laïque puisse juger en matière de doctrine fait de l'autorité de l'Église d'Angleterre en tant qu'ecclesia docens un sujet de risée, et la disqualifie de sa prétention à être une branche de l'Église catholique: 'If, in its Divine office it should fail, what remains?' écrit-il à Samuel Wilberforce. Dans une lettre du 20 février 1850, il lui explique que son dernier voyage en Italie n'a pas eu d'influence sur son évolution religieuse; il s'est tenu à l'écart des catholiques anglais et, si ce n'est avec trois ou quatre Romains, il n'a jamais discuté de points litigieux de théologie; c'est l'Église d'Angleterre qui achève de se déconsidérer à ses yeux :

And first let me say that although closer and longer experience of the Church abroad has convinced me of many things and made me to know by certain knowledge facts I never knew before, yet I can say with the fullest certainty a man may use in speaking of himself that my last visit to Italy has in no way weakened the grounds of my abiding in the English Church [...] I can therefore say that up to my return from Italy no increase of difficulty had arisen. Since then the care of the See of Hereford and of this appeal has seriously disturbed me by rendering all but untenable the grounds on which I have been resting. ${ }^{63}$

19 Ses préjugés à l'égard du catholicisme n'ont pas résisté à l'épreuve des faits ; ils se sont dissipés au fil de ses séjours sur le continent, et il a peu à peu résolu par l'étude et la pratique du sacerdoce les difficultés qui le préoccupaient depuis très longtemps. Ce sont ses doutes concernant l'Église d'Angleterre qui se sont accrus : l'affaire Hampden, puis l'affaire Gorham viennent de lui démontrer que son Église, qu'il avait cru divine, 
n'est qu'une société humaine, née du jugement privé et établie par le pouvoir civil. Le tollé que soulève dans l'Église anglicane le rétablissement de la hiérarchie catholique en Angleterre en septembre 1850 va lui donner le coup de grâce. L'Église anglicane, qui ne s'est pas émue de voir les questions de doctrine tranchées par un tribunal läque, s'enflamme avec une belle unanimité et une grande violence contre "l'agression papale ».

Le 22 novembre, après avoir une dernière fois réuni son clergé, qui entend protester contre ce rétablissement, Henry Edward Manning démissionne : il quitte sa paroisse de Lavington le 3 décembre, et résigne son bénéfice devant notaire en mars 1851. Reçu dans l'Église catholique le 6 avril en même temps que son ami James Hope, il reçoit les ordres mineurs le 30, avant d'être ordonné prêtre le 14 juin, trois ans presque jour pour jour après son retour de Rome. Ainsi prend fin le 'lustrum afflictionis et lacrymarum' qu'il évoquera plus tard dans une lettre à Gladstone ${ }^{64}$.

\section{NOTES}

1. Lettre à John Anderdon (5 avril 1831), in Edmund Sheridan PURCELL, Life of Cardinal Manning, Archbishop of Westminster, 2 vols., London \& New York: Macmillan, 1896, vol. 1, pp. 72-73.

2. Robert GRAY, Cardinal Manning: A Biography, London: Weidenfeld \& Nicolson, 1985, p. 33.

3. Stanley ROMER, Cardinal Manning as Presented in his own Letters and Notes, London: Elliot Stock, 1896, p. 6.

4. Shane LESLIE, Henry Edward Manning: His Life and Labours, London, 1921, p. 37.

5. Lettre à John Anderdon, in PURCELL, op. cit., vol. 1, p. 82.

6. Francis de PRESSENSÉ, Le cardinal Manning, Paris : Perrin et Cie, 1896, p.135.

7. Robert Gray rapporte qu'il avait refusé une paroisse de 1700 habitants pour laquelle il ne se sentait pas assez de force, ni de santé. Voir GRAY, op. cit., p. 43.

8. Lettre à Samuel Wilberforce (Manning MSS Bod., c.656, fo.55).

9. Journal de H. E. Manning, in PURCELL, op. cit., vol. 1, p.112.

10. Lettre à Henry Wilberforce (novembre 1833), in David NEWSOME, The Convert Cardinals: Newman and Manning, London: John Murray, 1993.

11. James PEREIRO, Cardinal Manning: An Intellectual Biography, Oxford: Oxford University Press, 1998, p. 21.

12. Lettre d'Edward Twisleton à H. E. Manning (14 décembre 1832), in PURCELL, op. cit., vol. 1, p. 92.

13. Ibid., p. 125.

14. 'I feel the absolute need of full employment' (lettre à Samuel Wilberforce, in NEWSOME, op. cit., p. 117).

15. Kenneth L. Parker, 'Henry Manning and Neo-Ultramontanism: The Anglican Context for an Oxford Movement Convert's Faith in Papal Infallibility', in Kenneth L. Parker \& Michael J. G. Pahl (eds.), Authority, Dogma, and History: The Role of the Oxford Movement Converts in the Papal Infallibility Debates, Bethesda, MD: Academica Press, 2009, p. 98.

16. Lettre de Samuel Wilberforce à Charles Anderson (7 décembre 1838), in PURCELL, op. cit., vol. 1, p. 140, note 1. 
17. Pour tous ces détails, voir M. R. D. FOOT \& H. C. G. MATTHEWS (eds.), The Gladstone Diaries, 14 vols., Oxford : Clarendon Press, 1968-1994, vol. 2 (1833-1839).

18. Lettre de John Sterling à Richard Trench (16 mars 1839), in LESLIE, op. cit., p. 59.

19. Lettre du 14 juin 1839. L'intégralité de la correspondance entre Manning et Gladstone sera bientôt disponible dans Peter C. ERB (ed.), The Correspondence of Henry Edward Manning and William Ewart Gladstone, 1833-1891 (à paraître en mars 2013 aux Presses de l'Université d'Oxford).

20. LESLIE, op. cit, p. 57.

21. Newman lui répond le $1^{\mathrm{er}}$ septembre : 'I think that nothing but patience and dutifulness can keep us in the Church of England'(GRAY, op. cit., p. 78).

22. Arthur Wollaston HUTTON, Cardinal Manning, London: Methuen and Co., 1892, p. 27.

23. Lettre à Gladstone (1842), in GRAY, op. cit., pp. 92-93.

24. Lettre du 25 octobre 1843, in PEREIRO, op. cit., p. 54 et GRAY, op. cit., p. 93.

25. Lettre à Gladstone (27 octobre1845), in LESLIE, op.cit., p. 65.

26. Lettre à Newman (21 décembre 1843), in Oratory of St. Philip Neri (ed.), Correspondence of John Henry Newman with John Keble and Others, 1839-1845, London \& New York: Longmans \& Green, 1917, p. 291.

27. Stewart J. BROWN, The National Churches of England, Ireland and Scotland, Oxford: Oxford University Press, 2001, p. 394.

28. ERB, op. cit. (lettre du 13 juillet 1844).

29. LESLIE, op. cit., p. 62.

30. Lettre du 13 juin 1844 (Manning MSS Bod., c.658, fo. 28).

31. ERB, op. cit. (lettre du 14 octobre 1844).

32. Henry Edward Manning, A Charge Delivered at the Ordinary Visitation of the Archdeaconry of Chichester in July, 1845, London: John Murray, 1845, pp. 51-52.

33. BROWN, op. cit., p. 395.

34. ERB, op. cit. (lettre du 14 août 1845).

35. Le Dr M. H. Luscombe, ancien aumônier de l'ambassade britannique à Paris, consacré évêque en 1825, était chargé par l'évêque de Londres de superviser les aumôneries du continent.

36. Détruit à la révolution, le séminaire a été reconstruit en 1820 , et les sulpiciens s'y sont réinstallés en 1826.

37. Voir A daughter of the Venerable Mother Pelletier, Sister Mary of the Sacred Heart Ryder, London: Convent of the Good Shepherd, 1902.

38. ERB, op. cit. (lettre à Catherine Gladstone, 19 octobre 1845).

39. Lettre à Robert Wilberforce, in NEWSOME, op. cit., p. 171 et PEREIRO, op. cit., p. 68.

40. PURCELL, op. cit., vol. 1, p. 329. Voir également Paul THUREAU-DANGIN, La Renaissance catholique en Angleterre au XIXe siècle, 2 vols., $2^{\text {nde }}$ ed., Paris : Librairie Plon, 1903, vol. 2, p. 129.

41. PEREIRO, op. cit., p. 70 (15 mai 1846).

42. Les chapitres XVII et XVIII du premier volume de la biographie de Purcell portent cette appellation.

43. Lettre du 16 juin 1847, in PURCELL, op. cit., vol. 1, pp. 468-469 et Alphonse CHAPEAU, « La Vie anglicane de Manning ", thèse de Doctorat d'État soutenue à Paris en 1955, pp. 545-547 (traduction française).

44. CHAPEAU, op. cit. (lettre du 22 avril 1847).

45. Ouverte quelques mois plus tôt, la chapelle de Fasque devait être consacrée le 26 juin 1847.

46. PURCELL, op.cit., vol. 1, p. 341.

47. Journal de H. E. Manning (entrée du 5 juillet 1847), in GRAY, op. cit., p. 119.

48. Alphonse CHAPEAU, 'Manning the Anglican', in Fitzsimons (ed.), Manning: Anglican and Catholic, London: The Catholic Book Club, 1951, p. 23.

49. ERB, op. cit. (lettre à Gladstone du 18 août 1847).

50. PURCELL, op. cit., vol. 1, p. 359; GRAY, op. cit., p. 120. 
51. Lettre à Gladstone (20 janvier 1848), in LESLIE, op.cit., p. 82.

52. Ibid.

53. Lettre de Florence Nightingale à son père (28 janvier 1848), in Lynn McDonald (ed.), Florence Nightingale's European Travels, Waterloo, Ontario, Canada : Wilfrid Laurier University Press, 2004, p. 237.

54. Lettre à Dodsworth (28 janvier 1848), in LESLIE, op. cit., p. 83. Les Passionistes sont les membres de la Congrégation de la Passion de Jésus-Christ, ordre catholique fondé en Italie en 1720.

55. Lettre de Newman à E. Coleridge (16 octobre 1844), in Louis BOUYER, Newman. Sa vie. Sa spiritualité, Paris : Éditions du Cerf, 1952, p. 298.

56. ERB, op. cit. (lettre du 3 avril 1848).

57. LESLIE, op. cit., p. 84. Les écrits de Ventura, qui justifiait, entre autres choses, l'insurrection irlandaise, seront bientôt mis à l'index.

58. Ibid., p. 85 ; NEWSOME, op. cit., p. 180.

59. Le Dr R. D. Hampden, dont la doctrine a été censurée à deux reprises par l'université d'Oxford, vient d'être nommé évêque par le premier ministre.

60. 'My opinions are what they were when I wrote to you from Rome. My charge is the case for the Church of England' (lettre à Moberly, in GRAY, op. cit., p. 128).

61. Lettre à Miss Hare (7 juin 1849), in PURCELL, op. cit., vol. 1, p. 431.

62. Lettre à Mary Wilberforce (Avent 1849), in LESLIE, op. cit., p. 88.

63. Lettre 81 (The Wilberforce Archives: Papers of Henry Edward Manning: Letters from Henry Edward Manning to Samuel Wilberforce).

64. ERB, op. cit. (lettre du 28 novembre 1862). L'expression est également rapportée dans LESLIE, op. cit., 'Preface', p. xi.

\section{RÉSUMÉS}

Pour expliquer sa conversion au catholicisme en 1851, le cardinal Henry Edward Manning (1808-1892) ne mentionne dans ses Notes Autobiographiques que son sacerdoce et ses lectures; ses voyages sur le continent, nombreux entre 1838 et 1848 , n'auraient-ils pas contribué tant soit peu à son évolution vers le catholicisme ? S'y rendait-il à la recherche de la santé ... ou de son salut?

To account for his conversion to Roman Catholicism in 1851, Cardinal Manning (1808-1892) in his Autobiographical Notes only mentions his priesthood and his readings; yet his numerous travels on the continent between 1838 and 1848 must have played an important part in his religious evolution. In other words, did he travel only for the sake of his health or for the sake of his soul?

\section{AUTEUR}

JACQUELINE CLAIS-GIRARD

Université d'Angers 